\title{
Joint Antenna Selection and Precoding Optimization for Small-Cell Network with Minimum Power Consumption
}

\author{
Qiang Sun, ${ }^{1,2}$ Xin Wang, ${ }^{1}$ Jue Wang, ${ }^{1,3}$ and Chen $\mathrm{Xu}{ }^{1}$ \\ ${ }^{1}$ The School of Electronic and Information, Nantong University, Nantong 226019, China \\ ${ }^{2}$ The National Mobile Communications Research Laboratory, Southeast University, Nanjing 210096, China \\ ${ }^{3}$ The Nantong Research Institute for Advanced Communication Technologies, Nantong 226019, China
}

Correspondence should be addressed to Chen Xu; xuchen@ntu.edu.cn

Received 7 July 2016; Accepted 15 March 2017; Published 28 March 2017

Academic Editor: Sotirios K. Goudos

Copyright (C) 2017 Qiang Sun et al. This is an open access article distributed under the Creative Commons Attribution License, which permits unrestricted use, distribution, and reproduction in any medium, provided the original work is properly cited.

\begin{abstract}
We focus on the power consumption problem for a downlink multiuser small-cell network (SCN) considering both the quality of service (QoS) and power constraints. First based on a practical power consumption model taking into account both the dynamic transmit power and static circuit power, we formulate and then transform the power consumption optimization problem into a convex problem by using semidefinite relaxation (SDR) technique and obtain the optimal solution by the CVX tool. We further note that the SDR-based solution becomes infeasible for realistic implementation due to its heavy backhaul burden and computational complexity. To this end, we propose an alternative suboptimal algorithm which has low implementation overhead and complexity, based on minimum mean square error (MMSE) precoding. Furthermore, we propose a distributed correlation-based antenna selection (DCAS) algorithm combining with our optimization algorithms to reduce the static circuit power consumption for the SCN. Finally, simulation results demonstrate that our proposed suboptimal algorithm is very effective on power consumption minimization, with significantly reduced backhaul burden and computational complexity. Moreover, we show that our optimization algorithms with DCAS have less power consumption than the other benchmark algorithms.
\end{abstract}

\section{Introduction}

Power consumption has increased considerably with the explosive rise of mobile data traffic demand over the past decade. Base stations have to consume much power to transmit a large amount of data traffic to meet the higher quality of service $(\mathrm{Q} o \mathrm{~S})$ required by the users. As pointed out in [1], base stations consume much more than sixty percent of the total power in the cellular networks. However, this is not in conformity with the lower power consumption and higher energy efficiency (EE) advocated by the green communications.

Spurred by growing environmental and economic concerns about how to sustain the exponential traffic growth, it is important to design energy saving wireless networks. The small-cell network (SCN) and massive MIMO are recognized as the key technologies for the decrease of power consumption because they have a great potential to enhance the EE
$[2,3]$. However, massive MIMO improves the EE, but at the cost of deploying more hardware infrastructure which means high power consumption. Besides, SCN achieves higher EE than massive MIMO in whatever crowded or sparse areas [4]. Unlike massive MIMO, SCN consists of a number of smallcell access points (SAPs), where each SAP is connected to a central processing unit (CPU) through a limited-capacity backhaul. SCN has less propagation losses and higher spatial reuse due to the short access distance provided [5], thereby resulting in higher EE.

So far, methods of improving the SCN's EE have been focused on in many related investigations such as communication mode [6], hardware improvement [7], and network deployment $[8,9]$. All the above literature provides comprehensive insights into how the EE can be improved, while the optimization for power consumption minimization of the SCN has also been well investigated in [1012]. However, the optimization methods in [10-12] are all 
based on coherent joint transmission, which requires full channel state information (CSI) of all jointly processed SAPs, a strict synchronization across the SAPs, and large backhaul capacity for information exchange [13]. Since the overhead of exchanging all information and computational complexity of joint processing is usually prohibitive for practical implementations, it is not suitable for coherent joint transmission in the SCN. Facing these challenges, we aim at designing efficient transmission schemes on the coordination and loosening the backhaul requirements for the $\mathrm{SCN}$.

In addition, it is well known that antenna selection can significantly reduce the complexity and power consumption to improve the spectral efficiency (SE) [14] and the EE [15, 16], which has been extensively studied for MIMO systems. However, most of these previous works for enhancing the SE and the EE ignored the fact that increasing the number of antennas is not always the best choice because of more power consumed in the circuits part [17]. Therefore, we believe that antenna selection is also an efficient approach to further reduce power consumption for the SCN.

To the best knowledge of the authors, the power consumption optimization, which combines the antenna selection and transmission scheme design for the SCN, is not presented before. Therefore, it is attractive to analyze and optimize this issue. In this work, we aim to minimize the power consumption under the quality of service (QoS) constraint per user and the power constraint per antenna of each SAP. First, we transform the initial power consumption minimization problem into a convex problem by using semidefinite relaxation (SDR) to obtain the system power consumption minimization and find the relationship among power consumption, the number of users, and the number of antennas per SAP. However, we know that it is difficult for this optimal algorithm to implement precoding in real-time due to heavy backhaul burden and high computational complexity when the number of SAPs is large. Then we propose a suboptimal algorithm which is on the basis of noncoherent joint transmission, for example, multi-SAP minimum mean square error (MMSE) precoding, to reduce the backhaul overhead and computational complexity. In addition, SAPs equipped with redundant antennas would consume a large amount of power consumption. In this case, we propose joint antenna selection and precoding optimization algorithms to further reduce power consumption. The simulation results show that there exists a small performance gap between the optimal algorithm and our proposed suboptimal algorithm. Moreover, our optimization algorithms with distributed correlation-based antenna selection (DCAS) are effective ways to minimize power consumption when the number of antennas is larger than the number of users per SAP.

The rest of this paper is organized as follows. In Sections 2 and 3, we present the system model and formulate the power consumption minimization problem, respectively. In Section 4, we propose a low overhead and complexity suboptimal algorithm. In Section 5, we propose the DCAS algorithm to further reduce the power consumption. The simulation results are presented in Section 6. Finally, the paper is concluded in Section 7.
Notation. Capital and small bold letters represent matrices and vectors, respectively; $\mathbf{A}^{H}$ and $\mathbf{A}^{T}$ stand for conjugate transpose and transpose of matrix $\mathbf{A}$, respectively. $\mathbb{C}^{M \times N}$ is the set of complex matrix with $M$ rows and $N$ columns, $\operatorname{tr}(\mathbf{A})$ is the trace of matrix $\mathbf{A}, \mathbf{I}$ is an identity matrix, and $\langle\mathbf{a}, \mathbf{b}\rangle$ denotes correlation of $\mathbf{a}$ and $\mathbf{b} . \mathscr{C} \mathscr{N}(\cdot, \cdot)$ stands for a multivariate circularly symmetric complex Gaussian distribution and we use $\|\cdot\|$ and $|\cdot|$ to denote the Euclidean norm and absolute value, respectively. The basic notations are given in the Notations.

\section{System Model}

As shown in Figure 1, we consider a downlink multiuser SCN consisting of $S$ small cells, where each small cell deploys a SAP with $N_{t}$ antennas and all the SAPs are connected to the CPU through the high-speed backhaul, via which some critical information, such as the CSI, is shared among all SAPs. There are $K$ single-antenna users simultaneously being served on the same frequency band. We assume the following aspects for the SCN:

(i) Constant User Number. Each SAP serves $K_{r}\left(\leq N_{t}\right)$ users and the number of users being served stays constant in each small cell.

(ii) Uniform Network Topology. SAPs are deployed uniformly and randomly in the SCN and the users are distributed uniformly and randomly in the coverage area of each SAP.

(iii) Perfect CSI. Perfect CSI is globally available at all SAPs and users.

Conventionally, each user is associated with only one SAP. Named as single-SAP transmission, it means that each SAP only serves the users in its own coverage area. However, in this paper, we assume a user can be associated with multiple SAPs and consider noncoherent multi-SAP transmission, which is much less complicated to be implemented in practice as compared to the coherent joint transmission which requires strict phase-synchronization [18]. Under noncoherent multiSAP transmission, each user can be associated by multiple SAPs but the information flow is encoded and transmitted independently at every SAP. In this way, it is able to strike a compromise between low implementation complexity (e.g., the single-SAP transmission) and good performance (e.g., the coherent joint transmission); thereby the noncoherent multi-SAP transmission can be attractive for practical system setups.

The channel vector between SAP $j$ and user $k$ is denoted by $\mathbf{h}_{k j} \in \mathbb{C}^{N_{t} \times 1}$, modeled as

$$
\mathbf{h}_{k j}=\sqrt{\beta_{k j}} \mathbf{g}_{k j},
$$

where $\mathbf{g}_{k j}$ is the small scale fading component whose elements are independent and identically distributed (i.i.d.) complex Gaussian random variables with zero-mean and unit variance and $\beta_{k j}$ represents the large scale fading including shadow fading, path loss, and penetration loss [3]. 


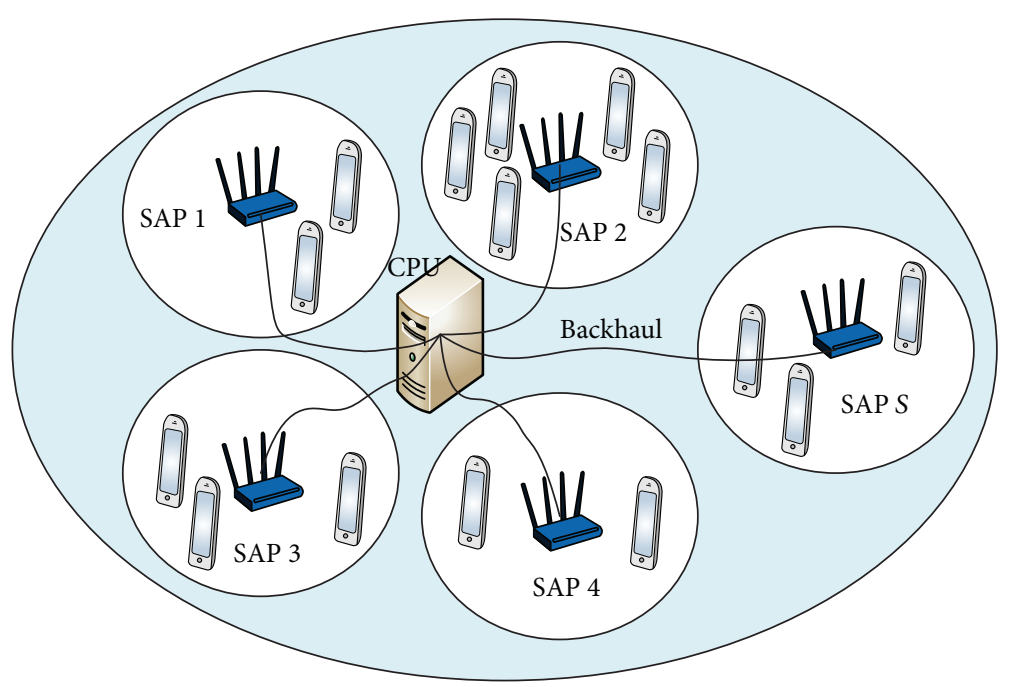

FIGURE 1: The system model of SCN.

The received signal at user $k$ is given by

$$
y_{k}=\sum_{j=1}^{S} \mathbf{h}_{k j}^{H} \mathbf{x}_{j}+n_{k},
$$

where $n_{k} \sim \mathscr{C} \mathscr{N}\left(0, \sigma_{k}^{2}\right)$ is the circularly symmetric complex Gaussian noise. At SAP $j$, the transmitted signal $\mathbf{x}_{j}$ is given by

$$
\mathbf{x}_{j}=\sum_{k=1}^{K} \mathbf{w}_{k j} f_{k j}, \quad j=1, \ldots, S,
$$

where $f_{k j} \in \mathscr{C} \mathscr{N}(0,1)$ is the transmitted signal from SAP $j$ to user $k$ and $\mathbf{w}_{k j} \in \mathbb{C}^{N_{t} \times 1}$ is the precoding vector, which is to be optimized to minimize the power consumption in the next section.

\section{Power Consumption Optimization}

The goal of our work is to minimize the power consumption in the considered SCN and show how the result is affected by the system parameters. We first formulate the power consumption minimization problem as follows. Unlike most ideal power consumption models where the circuits consumption is ignored, in this work, we consider a practical power consumption model which consists of two terms, respectively, corresponding to the transmitted power and the cost of circuits $[19,20]$ and denoted by $P_{1}$ and $P_{2}$ as follows:

$$
\begin{aligned}
& P_{1}=\sum_{j=1}^{S} \rho_{j} \sum_{k=1}^{K}\left\|\mathbf{w}_{k j}\right\|^{2}, \\
& P_{2}=\sum_{j=1}^{S} \alpha_{j} N_{t},
\end{aligned}
$$

where $\rho_{j} \geq 1$ and $\alpha_{j} \geq 0$ are parameters describing the inefficiency of the power amplifier at SAP $j$ and the power dissipation in the circuits of each antenna, respectively. The objective is to minimize $P_{1}+P_{2}$, under two practical constraints stated in the following.

(1) The power constraint per antenna of each SAP:

$$
\sum_{k=1}^{K} \mathbf{w}_{k j}^{H} \mathbf{D}_{j g} \mathbf{w}_{k j} \leq q_{j}, \quad \forall j, g,
$$

where $\mathbf{D}_{j g}$ is a positive semidefinite weighting matrix whose $g$ th diagonal elements are one and zero elsewhere. The weighting matrix can describe any combination of perantenna constraints. $q_{j}$ is fixed power constraint for each antenna at SAP $j$, defined in $\mathrm{mW}$.

(2) The QoS constraint per user (defined as the information rate in bits/s/Hz):

$$
\log _{2}\left(1+\operatorname{SINR}_{k}\right) \geq \gamma_{k}, \quad \forall k,
$$

where $\gamma_{k}$ is a fixed QoS target and the signal-to-interferenceand-noise ratio of user $k$ is given by

$$
\operatorname{SINR}_{k}=\frac{\sum_{j=1}^{S}\left|\mathbf{h}_{k j}^{H} \mathbf{w}_{k j}\right|^{2}}{\sum_{i=1, i \neq k}^{K} \sum_{j=1}^{S}\left|\mathbf{h}_{k j}^{H} \mathbf{w}_{i j}\right|^{2}+\sigma_{k}^{2}} .
$$

The first and second part of the denominator in (8) are interference and noise, respectively.

To minimize the power consumption of the system, the optimization problem is formulated as

$$
\begin{aligned}
\min _{\mathbf{w}_{k j}, \forall k, j} & P_{1}+P_{2} \\
\text { s.t. } & \log _{2}\left(1+\operatorname{SINR}_{k}\right) \geq \gamma_{k}, \quad \forall k \\
& \sum_{k=1}^{K} \mathbf{w}_{k j}^{H} \mathbf{D}_{j g} \mathbf{w}_{k j} \leq q_{j}, \quad \forall j, g .
\end{aligned}
$$

Due to the fact that $P_{2}$ is not related to $\mathbf{w}_{k j}$ in expression (5), $P_{2}$ is considered as a static part which is mainly 
determined by the number of antennas. While $P_{1}$ is dynamic, problem (9) can be solved if we make $P_{1}$ minimum while satisfying the QoS and power constraints. However, the constraints are not convex so that the CVX cannot work on problem (9).

To solve this problem, we reformulate (9) as a convex optimization problem by using SDR method. According to the definition in (7), we have

$$
\operatorname{SINR}_{k} \geq 2^{\gamma_{k}}-1 \triangleq \bar{\gamma}_{k}
$$

Plugging (8) into (10) yields

$$
\frac{1}{\bar{\gamma}_{k}} \sum_{j=1}^{S}\left|\mathbf{h}_{k j}^{H} \mathbf{w}_{k j}\right|^{2}-\sum_{i=1, i \neq k}^{K} \sum_{j=1}^{S}\left|\mathbf{h}_{k j}^{H} \mathbf{w}_{i j}\right|^{2} \geq \sigma_{k}^{2},
$$

which is alternatively expressed as

$$
\begin{aligned}
& \frac{1}{\bar{\gamma}_{k}} \sum_{j=1}^{S}\left|\mathbf{h}_{k j}^{H} \mathbf{w}_{k j}\right|^{2}-\left(\sum_{i=1}^{K} \sum_{j=1}^{S}\left|\mathbf{h}_{k j}^{H} \mathbf{w}_{i j}\right|^{2}-\sum_{j=1}^{S}\left|\mathbf{h}_{k j}^{H} \mathbf{w}_{k j}\right|^{2}\right) \\
& \quad \geq \sigma_{k}^{2} .
\end{aligned}
$$

Then, we define the positive semidefinite matrix

$$
\mathbf{V}_{k j}=\mathbf{w}_{k j} \mathbf{w}_{k j}^{H} \geq 0
$$

with $\operatorname{rank}\left(\mathbf{V}_{k j}\right) \leq 1$.

Using the above results, we obtain the inequality

$$
\sum_{j=1}^{S} \mathbf{h}_{k j}^{H}\left(\left(1+\frac{1}{\bar{\gamma}_{k}}\right) \mathbf{V}_{k j}-\sum_{i=1}^{K} \mathbf{V}_{i j}\right) \mathbf{h}_{k j} \geq \sigma_{k}^{2} .
$$

In addition, we can further relax the rank constraint $\operatorname{rank}\left(\mathbf{V}_{k j}\right) \leq 1$. The constraint can be removed with definitely satisfying the optimality as before [21]. Now (9) can be rewritten as

$$
\begin{aligned}
\min _{\mathbf{V}_{k j} \geq 0, \forall k, j} & \sum_{j=1}^{S} \rho_{j} \sum_{k=1}^{K} \operatorname{tr}\left(\mathbf{V}_{k j}\right)+P_{2} \\
\text { s.t. } & \sum_{k=1}^{K} \operatorname{tr}\left(\mathbf{D}_{j g} \mathbf{V}_{k j}\right) \leq q_{j}, \quad \forall j, g \\
& \sum_{j=1}^{S} \mathbf{h}_{k j}^{H}\left(\left(1+\frac{1}{\bar{\gamma}_{k}}\right) \mathbf{V}_{k j}-\sum_{i=1}^{K} \mathbf{V}_{i j}\right) \mathbf{h}_{k j} \geq \sigma_{k}^{2},
\end{aligned}
$$

$\forall k$.

Thus we can transform the initial power consumption minimization problem (9) into a convex problem (15), which can be solved by using CVX [22].

\section{Proposed Suboptimal Algorithm}

Solving the optimization problem in (15) requires centralized processing, for which the CSI of all SAP-user links should be known at the CPU. This induces large backhaul overhead and high computational complexity, which cannot be ignored especially when $S$ becomes large. In this section, we propose a suboptimal algorithm, which first alleviates the backhaul overhead by designing the multi-SAP MMSE precoding. Then, we transform (9) into a convex power allocation problem, reducing the computational complexity. The details of our proposed algorithm are shown as in the following steps.

\section{Proposed Suboptimal Algorithm}

Step 1. Each SAP computes its MMSE precoding vector; for example, SAP $j$ designs the precoding vector for user $k$ given by

$$
\mathbf{e}_{k j}=\frac{\mathbf{w}_{k j}}{\left\|\mathbf{w}_{k j}\right\|}, \quad \forall j, k,
$$

where $\mathbf{w}_{k j}=\left(\sum_{i=1}^{K} \mathbf{h}_{i j} \mathbf{h}_{i j}^{H}+\tau \mathbf{I}\right)^{-1} \mathbf{h}_{k j}$ and $\tau=K \sigma_{k}^{2}$ in this paper; then each SAP can easily obtain the following parameters.

$$
\begin{gathered}
m_{i k j}=\left|\mathbf{h}_{i j}^{H} \mathbf{e}_{k j}\right|^{2}, \quad \forall i, k, \\
D_{j g k}=\mathbf{e}_{k j}^{H} \mathbf{D}_{j g} \mathbf{e}_{k j}, \quad \forall g, k .
\end{gathered}
$$

Step 2. The scalar parameters $m_{i k j}$ and $D_{j g k}$ computed in (17) are sent back to the CPU to solve the following convex optimization problem [21].

$$
\begin{aligned}
\min _{p_{k j} \geq 0, \forall k, j} & \sum_{j=1}^{S} \rho_{j} \sum_{k=1}^{K} p_{k j}+P_{2} \\
\text { s.t. } \quad & \sum_{k=1}^{K} D_{j g k} p_{k j} \leq q_{j}, \quad \forall j, g \\
& \sum_{j=1}^{S} p_{k j} m_{k k j}\left(1+\frac{1}{\bar{\gamma}_{k}}\right)-\sum_{i=1}^{K} p_{i j} m_{k i j} \geq \sigma_{k}^{2},
\end{aligned}
$$

Step 3. The result of power allocation optimized in (18) by the CPU is sent to each SAP; for example, $p_{k j}^{*}$ is informed to SAP $j$.

Step 4. Each SAP transmits the data to the served users with the suboptimal precoding vector

$$
\mathbf{w}_{k j}=\sqrt{p_{k j}^{*}} \mathbf{e}_{k j}, \quad \forall k .
$$

We observe that the precoding vector is obtained by expression (16) rather than optimization (15), which means the distributed precoding design has been done by each SAP before the power allocation problem (18) is optimized. As a result, the CPU needs little backhaul overhead to complete the power allocation. Compared with the optimal algorithm which has to share complete CSI of each user, the proposed suboptimal algorithm exchanges only two scalars, that is, $m_{i k j}$ and $D_{j g k}$, between each SAP and the CPU. Moreover, 
the optimization variable is the vector $\mathbf{w}$ in the optimal algorithm, while it is the scalar $p$ in our proposed suboptimal algorithm. All of these point out that the backhaul overhead and computational complexity of the proposed suboptimal algorithm can be significantly reduced as compared to the original problem in (15).

\section{Antenna Selection Algorithm}

In this section, we show that the system power consumption can be further reduced by combining the proposed precoding optimization with properly designed antenna selection algorithm. Note that when the circuit power consumption is not considered in the analysis, having more antennas will always lead to lower power consumption for the reason that it provides more degree of freedom. However, as we are considering the practical power consumption model where the circuit power consumption cannot be ignored, increasing the number of antennas (i.e., degree of freedom) comes at a cost of consuming more circuit power. Therefore, it may be not optimal to use all $N_{t}$ antennas at every SAP for transmission, and there always exists an optimal number of antennas to minimize the total power consumption.

In general, antenna selection algorithm can be formulated as a combinatorial optimization problem, which can be solved by exhaustive search (ES) method, but leading to a prohibitive computational burden even for a small number of antennas and users. The simplest antenna selection algorithm is known as the random antenna selection (RAS) which has very low complexity but poor performance. Alternatively, in this work, we propose the DCAS algorithm. With this algorithm, each SAP individually selects the best $L$ out of $N_{t}$ antennas of its own before doing optimization algorithms. The channel matrix between SAP $j$ and its corresponding $K_{r}$ users is $\mathbf{H}_{j}=\left[\mathbf{h}_{1 j} \mathbf{h}_{2 j} \cdots \mathbf{h}_{k_{r}}\right]$ with $N_{t}$ rows and $K_{r}$ columns (it is shown by simulations that the optimal $L$ should be set equal to the number of served users $K_{r}$ in the corresponding small cell). The $a$ th and $b$ th row of the channel matrix $\mathbf{H}_{j}$ are denoted by $\left[\mathbf{H}_{j}\right]_{a}$ and $\left[\mathbf{H}_{j}\right]_{b}$, respectively. With these notations, the DCAS algorithm is described as in the following steps.

\section{DCAS Algorithm}

Step 1. $\forall a, b$, SAP $j$ computes the correlation $\left\langle\left[\mathbf{H}_{j}\right]_{a},\left[\mathbf{H}_{j}\right]_{b}\right\rangle$, $j=1,2, \ldots, S$.

Step 2. SAP $j$ chooses the correlation $\left\langle\left[\mathbf{H}_{j}\right]_{a},\left[\mathbf{H}_{j}\right]_{b}\right\rangle$ which is the largest, $j=1,2, \ldots, S$.

Step 3. If $\left\|\left[\mathbf{H}_{j}\right]_{a}\right\|^{2} \leq\left\|\left[\mathbf{H}_{j}\right]_{b}\right\|^{2}$, SAP $j$ deletes $\left[\mathbf{H}_{j}\right]_{a}$; otherwise, it deletes $\left[\mathbf{H}_{j}\right]_{b}, j=1,2, \ldots, S$.

Step 4. Repeat Steps 2 and 3 until $N_{t}-L$ rows are all deleted; then each SAP obtains a selected channel matrix $\widehat{\mathbf{H}}_{j}=\left[\widehat{\mathbf{h}}_{1 j} \widehat{\mathbf{h}}_{2 j} \cdots \widehat{\mathbf{h}}_{k_{r} j}\right]$ with $L$ rows and $K_{r}$ columns, where the channel vector between SAP $j$ and user $k$ is denoted by $\widehat{\mathbf{h}}_{k j} \in \mathbb{C}^{L \times 1}$.

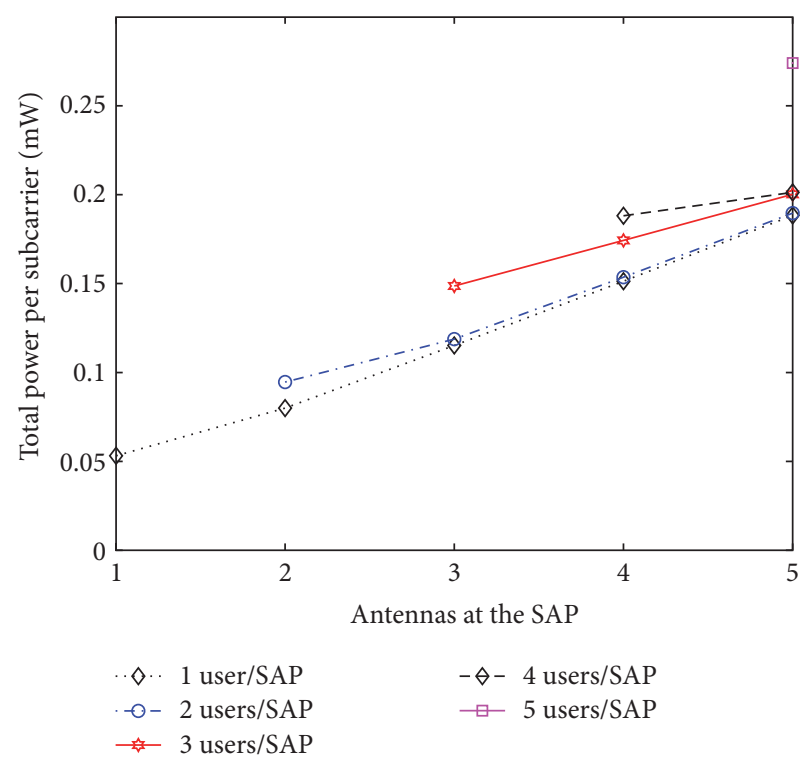

FIGURE 2: Total power consumption versus the number of antennas per SAP.

This algorithm is explained as follows: we select two rows in $\mathbf{H}_{j}$ with the highest correlation and eliminate the one with lower power and then repeat this process until $N_{t}-L$ rows are all deleted. After this antenna selection procedure, the dimension of the channel matrix is reduced from $N_{t} \times$ $K_{r}$ to $L \times K_{r}$. The resultant channel matrix will have rows with minimal correlation and maximal power; therefore a large capacity is expected for this dimension-reduced channel matrix.

\section{Simulation Results}

In this section, we give numerical simulations to evaluate the power consumption performance of our proposed precoding optimization and antenna selection algorithms. The simulation is implemented on the Matlab platform with the toolbox CVX. We set the number of antennas and users per SAP to be $N_{t} \in\{1,2,3,4,5\}$ and $K_{r} \in\{1,2,3,4,5\}$, respectively. The SAPs are deployed arbitrarily in the cell and the users are distributed uniformly and randomly in the area of each SAP as shown in Figure 1 (we find that the different SAP density scenarios will not affect the conclusion in Figure 2 through extensive simulations). Some of the parameters follow LTE standard; others are based on [23,24], as Table 1 shows.

In Figure 2, we show the total power consumption of the precoding optimization for different numbers of users per SAP when the QoS target is $2 \mathrm{bits} / \mathrm{s} / \mathrm{Hz}$. In ideal power consumption models, we generally believe that the power consumption decreases with the increasing number of antennas, while, in our practical model, the power consumption rises as the number of antennas increases. We find that no feasible solution can be obtained when the number of users is larger than the number of antennas per SAP due to QoS constraint per user. 
TABLE 1: Simulation parameters.

\begin{tabular}{lc}
\hline Parameters & Setting \\
\hline Inefficiency of power amplifiers & $\rho_{j}=19.23, \forall j$ \\
Circuit power dissipation per antenna & $\alpha_{j}=5.6 \mathrm{~mW}, \forall j$ \\
Per-antenna power constraint & $q_{j}=0.08 \mathrm{~mW}, \forall j$ \\
Small scale fading distribution & $\mathbf{g}_{k j} \sim \mathscr{C} \mathcal{N}\left(0, \mathbf{I}_{N_{t}}\right) \forall k, j$ \\
Path and penetration loss model between each SAP at distance $d(\mathrm{~km})$ & $148.1+37.6 \log _{10}(d) \mathrm{dB}$ \\
Path and penetration loss model in each small cell at distance $d(\mathrm{~km})$ & $127+30 \log _{10}(d) \mathrm{dB}$ \\
Noise variance & $\sigma_{k}^{2}=-127 \mathrm{dBm}, \forall k$ \\
Standard deviation of log-normal shadowing & $7 \mathrm{~dB}$ \\
\hline
\end{tabular}

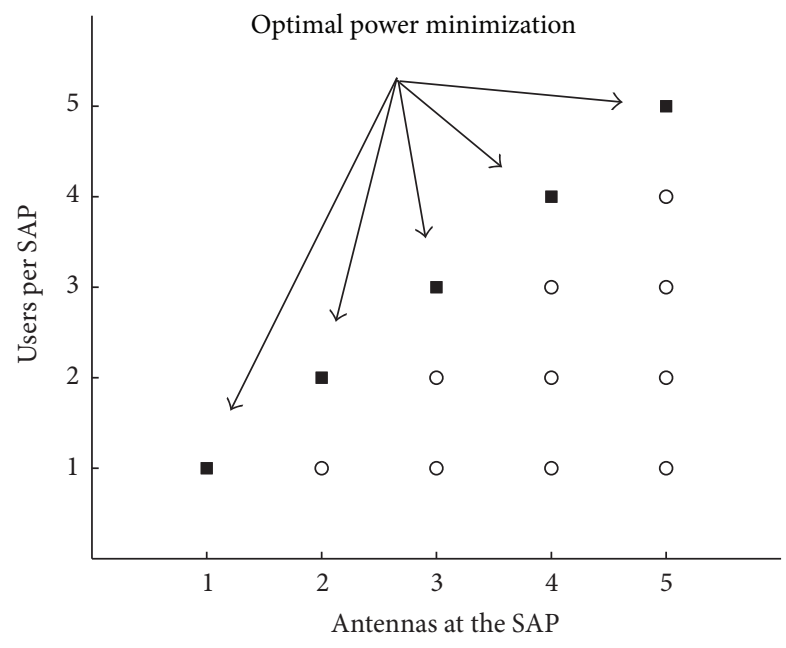

FIGURE 3: Distribution of solutions.

Figure 3 describes the distribution of solutions of the precoding optimization. Combining with Figure 2, we see that it is optimal to serve the user by only one SAP which is the nearest instead of all of the SAPs. It is interesting to observe that in our practical power consumption model, under the condition that the ratio of the number of antennas and the number of users is $1: 1$, the total power consumption of the SCN is globally minimal. This means that the SCN has the best performance when the number of selected antennas equals the number of served users per SAP. Motivated by the above conclusion, we will consider antenna selection to further reduce circuit power consumption for the SCN.

In the simulation of Figure 4 , we set $N_{t}=2$ and $K_{r}=2$ per SAP. Three schemes are compared: the optimal optimization algorithm, the suboptimal optimization algorithm, and the original scheme without any optimization. It is shown that without any antenna selection, the suboptimal optimization algorithm shows satisfactory performance as the gap between the suboptimal algorithm and the optimal algorithm is narrow and acceptable. It should be emphasized that although there is slight performance loss as compared with the optimal algorithm, the proposed suboptimal algorithm is able to greatly reduce the execution time consumption, as will be shown later.

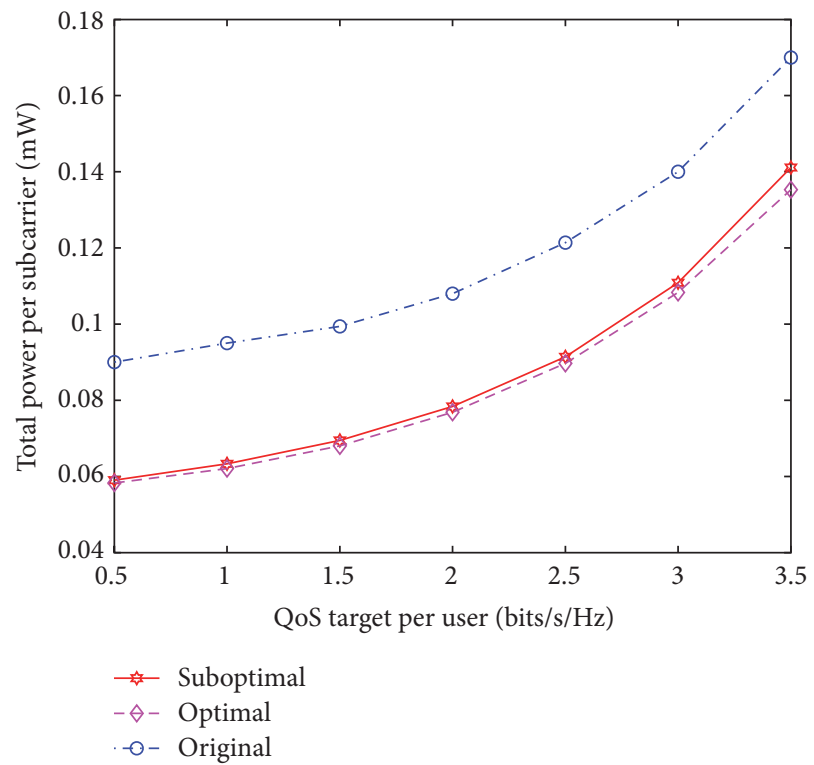

FIGURE 4: Total power consumption versus QoS target per user. Results are shown for different optimization algorithms.

Table 2 compares the execution times of simulation work between the optimal optimization algorithm and the suboptimal optimization algorithm (the execution time is measured under the following conditions: Matlab: R2014a version; CPU: Intel Core i7-4720HQ; RAM: 8 GB DDR3L). We set $S=3$ and $N_{t}=K_{r}$ in the simulation. The execution time shown in Table 2 describes the time used by the optimal and suboptimal algorithms for the different number of users. We find that the time used for the suboptimal algorithm is always in the scale of millisecond, which is much less than the time consumption of the optimal algorithm. With increasing number of users, the gap of the execution time between these two algorithms becomes larger. The comparison results show that the optimal optimization algorithm is time-consuming to be implemented in practice, especially for dense networks, where the number of users is always large. On the other hand, the proposed suboptimal algorithm provides a more efficient solution with negligible performance loss.

Figure 5 demonstrates the effectiveness of the antenna selection algorithm. We consider $N_{t}=5$ and $K_{r}=2$ per SAP. 
TABLE 2: Comparison of execution times used by the optimal and suboptimal algorithms.

\begin{tabular}{|c|c|c|c|c|c|}
\hline & $K_{r}=2$ & $K_{r}=4$ & $K_{r}=6$ & $K_{r}=8$ & $K_{r}=10$ \\
\hline Optimal algorithm & $69 \mathrm{~ms}$ & $200 \mathrm{~ms}$ & $680 \mathrm{~ms}$ & $2457 \mathrm{~ms}$ & $7000 \mathrm{~ms}$ \\
\hline Suboptimal algorithm & $32 \mathrm{~ms}$ & $60 \mathrm{~ms}$ & $88 \mathrm{~ms}$ & $118 \mathrm{~ms}$ & $150 \mathrm{~ms}$ \\
\hline
\end{tabular}

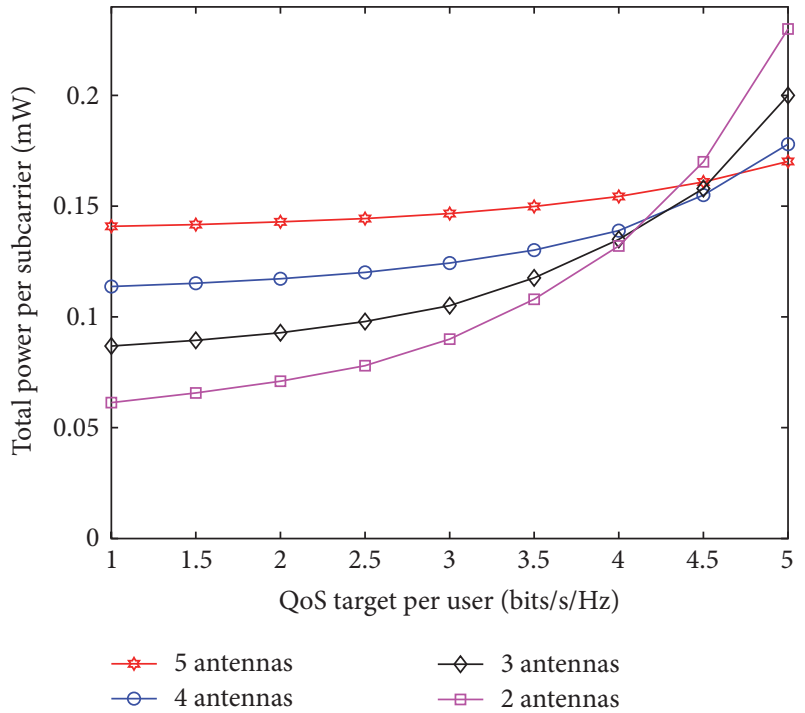

FIGURE 5: Total power consumption versus QoS target per user. Results are shown for DCAS algorithm when the number of antennas is no less than that of users.

Assume that five antennas are all available and we use DCAS algorithm to select two, three, four, and five of them to serve the two users, respectively. We can conclude that the total power consumption increases with the increasing number of selected antennas when the QoS target is below $3.5 \mathrm{bits} / \mathrm{s} / \mathrm{Hz}$. The total power consumption is minimal if we select the same number of antennas as the number of users before our optimization algorithm. The reason is that when the QoS constraint is in the range of IMT-Advanced requirement, $P_{2}$ is the major part of the total power consumption (the downlink SE of the IMT-Advanced requirement is up to 3 bits $/ \mathrm{s} / \mathrm{Hz}$ and $2.25 \mathrm{bits} / \mathrm{s} / \mathrm{Hz}$ for indoor usage. In this paper, we mainly consider the QoS target is below $3.5 \mathrm{bits} / \mathrm{s} / \mathrm{Hz}$, which meets the IMT-Advanced requirement [18]). In this case, the total power consumption depends mostly on the number of selected antennas. When the QoS target per user is larger than $3.5 \mathrm{bits} / \mathrm{s} / \mathrm{Hz}, P_{1}$ becomes to dominate the total power consumption to satisfy all the users' requirements, resulting in a better performance by selecting as many antennas as possible.

Figure 6 focuses on the total power consumption versus QoS target per user when the number of antennas is larger than that of users. We consider $N_{t}=3$ and $K_{r}=2$ per SAP and compare the following seven schemes:

(i) S1: The power consumption with optimal algorithm and DCAS algorithm.

(ii) S2: The power consumption with proposed suboptimal algorithm and DCAS algorithm.

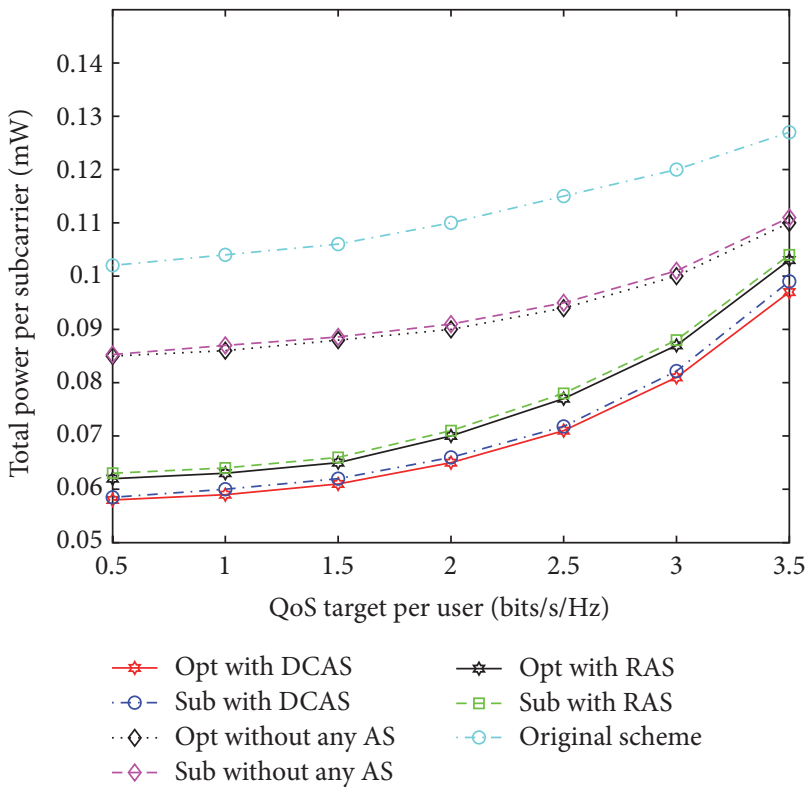

FIGURE 6: Total power consumption versus QoS target per user. Results are shown for different schemes.

(iii) S3: The power consumption with optimal algorithm but without any antenna selection.

(iv) S4: The power consumption with proposed suboptimal algorithm but without any antenna selection.

(v) S5: The power consumption with optimal algorithm and RAS algorithm.

(vi) S6: The power consumption with proposed suboptimal algorithm and RAS algorithm.

(vii) S7: The original power consumption without any optimization or antenna selection.

The comparison result demonstrates that the performance of our proposed suboptimal algorithm S4 is superior to the original scheme S7 but a little worse than the optimal algorithm S3. Table 3 shows the comparison of overhead and complexity between the optimal algorithm S3 and our proposed suboptimal algorithm $\mathrm{S} 4$. We see that the optimal algorithm has to exchange $2 S K N_{t}$ backhaul data in total, while only $2 S K$ parameters need to be exchanged in the proposed suboptimal algorithm, resulting in less backhaul overhead. The CPU has to compute the trace of matrices in the optimal algorithm, while the computation of our proposed suboptimal algorithm just involves scalars, yielding lower computational complexity.

In addition, Figure 6 shows the gap between the optimal algorithm and the suboptimal algorithm is rather small, 
TABLE 3: Comparison of total overhead and complexity.

\begin{tabular}{lcc}
\hline & Overhead & Complexity \\
\hline Optimal algorithm & $\mathcal{O}\left(2 S K N_{t}\right)$ & $\mathcal{O}\left(S K N_{t}{ }^{3}\right)$ \\
Suboptimal algorithm & $\mathcal{O}(2 S K)$ & $\mathcal{O}(S K)$ \\
\hline
\end{tabular}

so it is feasible to implement the suboptimal algorithm in practice rather than the optimal algorithm owing to its low backhaul overhead and computational complexity. Our proposed suboptimal algorithm will work well even with deploying more antennas or dense networks. Moreover, we see that our optimization algorithms with DCAS can further reduce the power consumption and have better performance than the other schemes with RAS. Based on the conclusions in this paper, our proposed suboptimal power consumption algorithm with DCAS is the most proper scheme to implement.

\section{Conclusion}

In this work, we investigated joint antenna selection and precoding design for the SCN to minimize the power consumption while maintaining QoS requirements of the users. We formulated the power consumption minimization problem based on a realistic power consumption model. Using SDR technique, the original nonconvex problem is converted to convex and efficiently solved by the CVX toolbox. To further reduce the backhaul overhead and computational complexity, we proposed a suboptimal algorithm based on noncoherent multi-SAP MMSE precoding, which requires scalar parameters (other than the complete CSI) being fed back to the CPU, only optimizing a simple power allocation problem. Simulation results showed that the proposed suboptimal algorithm greatly reduces the overhead and complexity without sacrificing much performance. Moreover, we demonstrated that the power consumption can be further reduced by combining our optimization algorithms with the DCAS algorithm.

\section{Notations}

K: $\quad$ The total number of users

$K_{r}: \quad$ The number of users per SAP

$S: \quad$ The number of SAPs

$N_{t}$ : The number of antennas per SAP

$n_{k}$ : The circularly symmetric complex Gaussian noise

$\mathbf{h}_{k j}$ : The channel vector between SAP $j$ and user $k$

$y_{k}$ : The received signal of user $k$

$\mathbf{x}_{j}$ : The transmitted signal from SAP $j$

$\mathbf{f}_{k j}$ : The information symbol from SAP $j$ to user $k$

$\mathbf{w}_{k j}$ : The precoding vector from SAP $j$ to user $k$

$\gamma_{k}, q_{j}$ : The QoS and power constraint target

$\sigma_{k}^{2}: \quad$ The noise variance

$\rho_{j}: \quad$ The inefficiency of the power amplifier at SAP $j$

$\alpha_{j}$ : The power dissipation in the circuits.

\section{Conflicts of Interest}

The authors declare that there are no conflicts of interest regarding the publication of this paper.

\section{Acknowledgments}

This work was supported by National Natural Science Foundation of China under Grant nos. 61501264, 61401240, and 61371111, the open research fund of National Mobile Communications Research Laboratory, Southeast University (no. 2015D02), Jiangsu Government Scholarship for Overseas Studies (JS-2014-206), the Doctoral Scientific Research Foundation of Nantong University under Grant 14B08, and Nantong University-Nantong Joint Research Center for Intelligent Information Technology under Grant no. 0702160101.

\section{References}

[1] Z. Hasan, H. Boostanimehr, and V. K. Bhargava, "Green cellular networks: a survey, some research issues and challenges," IEEE Communications Surveys and Tutorials, vol. 13, no. 4, pp. 524540, 2011.

[2] J. Hoydis, M. Kobayashi, and M. Debbah, "Green small-cell networks," IEEE Vehicular Technology Magazine, vol. 6, no. 1, pp. 37-43, 2011.

[3] E. Björnson, M. Kountouris, and M. Debbah, "Massive MIMO and small cells: improving energy efficiency by optimal softcell coordination," in Proceedings of the 20th International Conference on Telecommunications (ICT '13), pp. 1-5, IEEE, Casablanca, Morocco, May 2013.

[4] H. D. Nguyen and S. Sun, "Massive MIMO versus small-cell systems: spectral and energy efficiency comparison," https://arxiv .org/abs/1509.03998.

[5] Q. Sun, J. Wang, S. Jin, C. Xu, X. Gao, and K.-K. Wong, "Rate analysis and pilot reuse design for dense small cell networks," in Proceedings of the IEEE International Conference on Communications (ICC '15), pp. 2845-2850, London, UK, June 2015.

[6] I. Atzeni and M. Kountouris, "Full-duplex MIMO small-cell networks: performance analysis," in Proceedings of the IEEE Global Communications Conference (GLOBECOM '15), December 2015.

[7] T. Oh, Y.-G. Lim, C.-B. Chae, and Y. Lee, "Dual-polarization slot antenna with high cross-polarization discrimination for indoor small-cell MIMO systems," IEEE Antennas and Wireless Propagation Letters, vol. 14, pp. 374-377, 2015.

[8] C. Li, J. Zhang, and K. B. Letaief, “Throughput and energy efficiency analysis of small cell networks with multi-antenna base stations," IEEE Transactions on Wireless Communications, vol. 13, no. 5, pp. 2505-2517, 2014.

[9] E. Bjornson, L. Sanguinetti, and M. Kountouris, "Designing wireless broadband access for energy efficiency: are small cells the only answer?" in Proceedings of the IEEE International Conference on Communication Workshop (ICCW '15), pp. 136141, June 2015.

[10] A. Tölli, H. Pennanen, and P. Komulainen, "Decentralized minimum power multi-cell beamforming with limited backhaul signaling," IEEE Transactions on Wireless Communications, vol. 10, no. 2, pp. 570-580, 2011. 
[11] Y. Li, Y. Tian, and C. Yang, "Energy-efficient coordinated beamforming under minimal data rate constraint of each user," IEEE Transactions on Vehicular Technology, vol. 64, no. 6, pp. 2387-2397, 2015.

[12] S. Lakshminarayana, M. Assaad, and M. Debbah, “Transmit power minimization in small cell networks under time average QoS constraints," IEEE Journal on Selected Areas in Communications, vol. 33, no. 10, pp. 2087-2103, 2015.

[13] L. Sanguinetti, R. Couillet, and M. Debbah, "Large system analysis of base station cooperation for power minimization," https://arxiv.org/abs/1509.00731.

[14] A. F. Molisch, M. Z. Win, Y.-S. Choi, and J. H. Winters, "Capacity of MIMO systems with antenna selection," IEEE Transactions on Wireless Communications, vol. 4, no. 4, pp. 1759-1771, 2005.

[15] C. Jiang and L. J. Cimini, "Antenna selection for energy-efficient MIMO transmission," IEEE Wireless Communications Letters, vol. 1, no. 6, pp. 577-580, 2012.

[16] X. Zhou, B. Bai, and W. Chen, "Iterative antenna selection for multi-stream MIMO under a holistic power model," IEEE Wireless Communications Letters, vol. 3, no. 1, pp. 82-85, 2014.

[17] X. Zhou, B. Bai, and W. Chen, "Invited paper: antenna selection in energy efficient MIMO systems: a survey," China Communications, vol. 12, no. 9, pp. 162-173, 2015.

[18] H. Holma and A. Toskala, LTE Advanced: 3GPP Solution for IMT-Advanced, John Wiley \& Sons, 2012.

[19] S. Cui, A. J. Goldsmith, and A. Bahai, "Energy-constrained modulation optimization," IEEE Transactions on Wireless Communications, vol. 4, no. 5, pp. 2349-2360, 2005.

[20] D. W. K. Ng, E. S. Lo, and R. Schober, "Energy-efficient resource allocation in OFDMA systems with large numbers of base station antennas," IEEE Transactions on Wireless Communications, vol. 11, no. 9, pp. 3292-3304, 2012.

[21] E. Björnson and E. Jorswieck, "Optimal resource allocation in coordinated multi-cell systems," Foundations and Trends in Communications and Information Theory, vol. 9, no. 2-3, pp. 113381, 2013.

[22] CVX Research Inc, CVX: Matlab Software for Disciplined Convex Programming, Version 2.1 Beta, 2015, http://cvxr.com/cvx.

[23] M. A. Imran, E. Katranaras, and G. Auer, "Energy efficiency analysis of the reference systems, areas of improvements and target breakdown," ICT-EARTH Project, 2011.

[24] R. V. R. Kumar and J. Gurugubelli, "How green the LTE technology can be?" in Proceedings of the IEEE 2nd International Conference on Wireless Communication, Vehicular Technology, Information Theory and Aerospace \& Electronic Systems Technology (Wireless VITAE '11), pp. 1-5, Chennai, India, February 2011. 


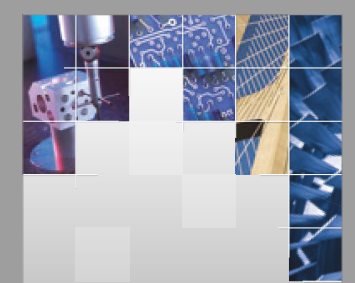

\section{Enfincering}
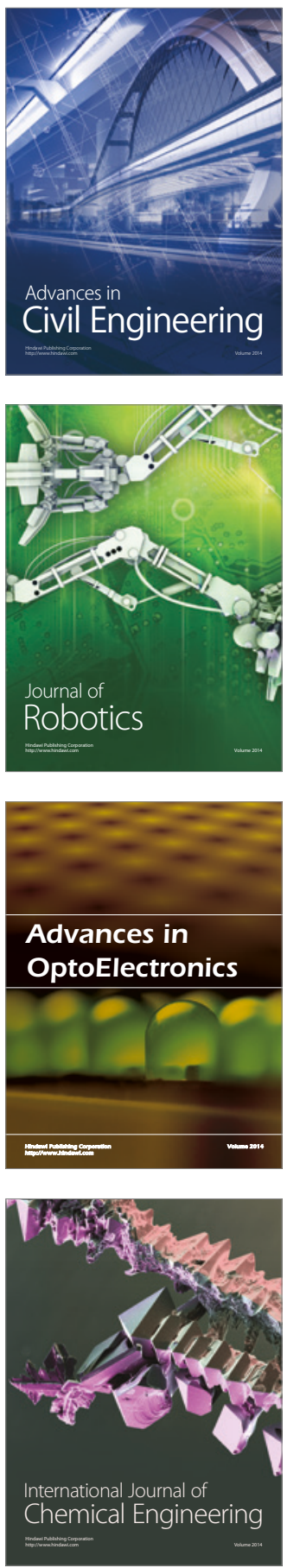

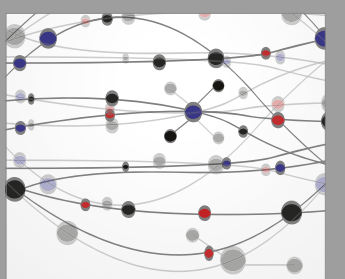

The Scientific World Journal

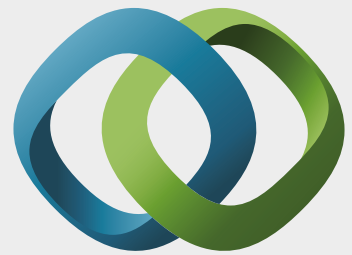

\section{Hindawi}

Submit your manuscripts at

https://www.hindawi.com
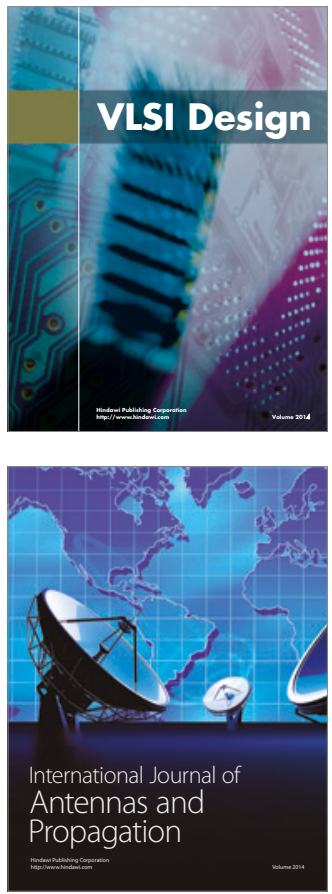

\section{Rotating}

Machinery
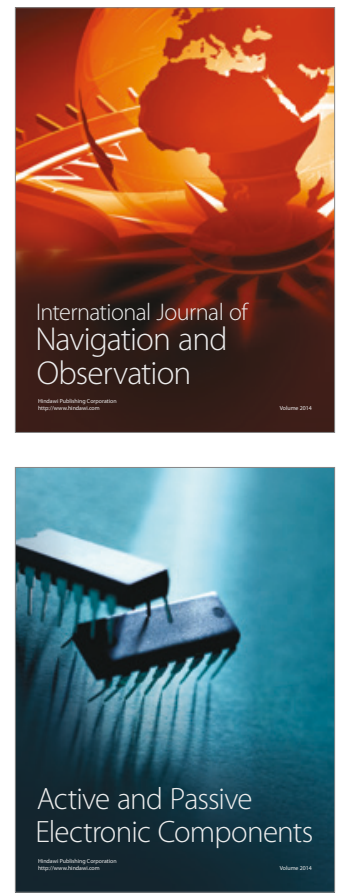
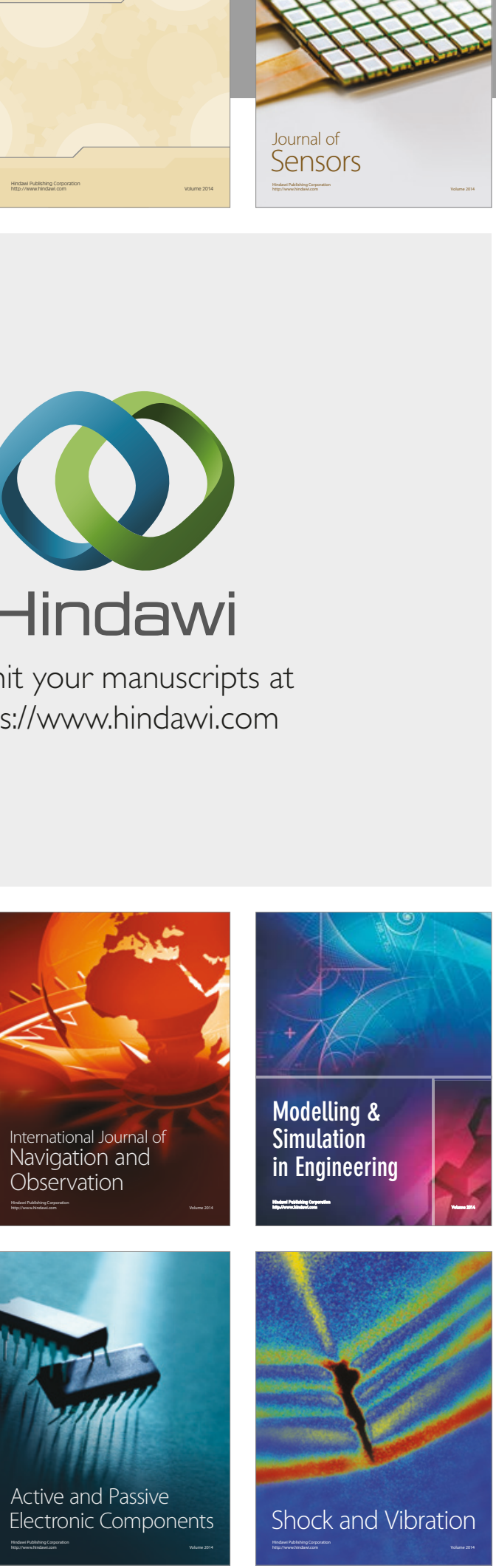
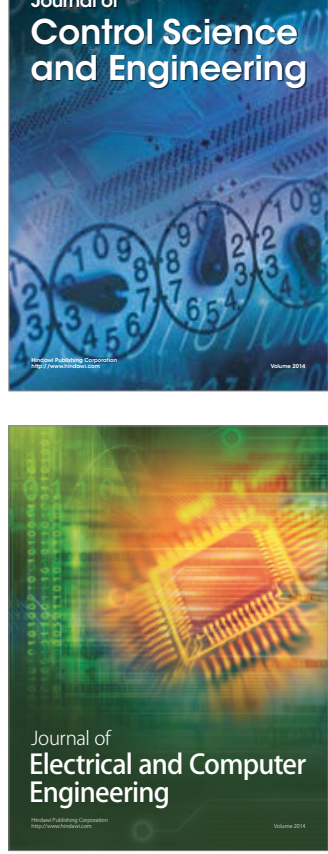

Distributed

Journal of

Control Science

and Engineering
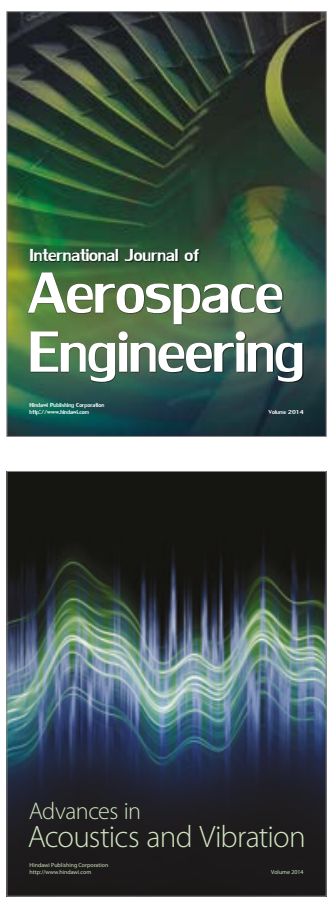

Sensor Networks 